\title{
Genetic and morphological evidence reveals the existence of a new family, genus and species of Echinorhynchida (Acanthocephala)
}

\author{
Paola E. Braicovich ${ }^{1}$, Ana L. Lanfranchi ${ }^{1}$, Marisa D. Farber ${ }^{2}$, Adriana E. Marvaldi ${ }^{3}$, José L. Luque ${ }^{4}$ and \\ Juan T. Timi ${ }^{1}$ \\ ${ }^{1}$ Laboratorio de Parasitología, Instituto de Investigaciones Marinas y Costeras (IIMyC), FCEyN, Universidad Nacional de Mar del \\ Plata-CONICET, Mar del Plata, Argentina; \\ ${ }^{2}$ Instituto de Biotecnología, Instituto Nacional de Tecnología Agropecuaria (INTA), Hurlingham, Buenos Aires, Argentina; \\ ${ }^{3}$ Instituto Argentino de Investigaciones de Zonas Aridas (IADIZA), CCT-CONICET Mendoza, Mendoza, Argentina; \\ ${ }^{4}$ Departamento de Parasitologia Animal, Universidade Federal Rural do Rio de Janeiro, Seropédica, Brazil
}

\begin{abstract}
Gymnorhadinorhynchus gen. n. is proposed to accommodate its type species, G. decapteri sp. n., a parasite of the marine fish Decapterus punctatus (Cuvier), caught from the coastal waters of Brazil. Gymnorhadinorhynchus decapteri sp. n. was morphologically most similar to species of two echinorhynchid families, the Rhadinorhynchidae and the Cavisomidae, particularly in the structure of the proboscis and the absence of somatic spines, respectively. This combination of morphological features made it difficult to assign our specimen to an extant family of the Acanthocephala. Therefore, in order to clarify the systematic placement of $G$. decapteri, a molecular phylogenetic analysis was performed based on the SSU and LSU rDNA and the mitochondrial cox 1 gene sequences obtained for the new taxon and other 26 acanthocephalan species. The results of parsimony and maximum likelihood analyses, using individual, combined and concatenated sequence data, consistently indicate that the specimens do not belong to any known family of the Echinorhynchida. Rather, G. decapteri represents a distinct lineage that is closely related to the Transvenidae, but distantly related to both the Rhadinorhynchidae and the Cavisomidae. Gymnorhadinorhynchidae fam. $\mathrm{n}$. is therefore erected. This newly described family can be distinguished from other families of Echinorhynchida by the combination of the following morphological characters: a proboscis cylindrical with 10 rows of 22-26 hooks, dorsoventral differences in proboscis hooks, basal hooks forming a ring and being abruptly larger than anterior hooks, absence of trunk spines and presence of four tubular cement glands. This combination, in addition to several molecular autapomorphies, justifies the erection of a new genus, Gymnorhadinorhynchus gen. $\mathrm{n}$., in order to accommodate this new species.
\end{abstract}

Keywords: Rhadinorhynchidae, Cavisomidae, Gymnorhadinorhynchidae fam. n., Gymnorhadinorhynchus gen. n., marine fish

This article contains supporting information (S1, S2). S1 - Nexus file (saved in Word as Text Only) with the combined aligned data, file name: NEX str18S\&28S cox1_11feb2014.txt, online at http://folia.paru.cas.cz/suppl/2014-4-377b.txt; S2 - Colour Word file with the annotated structure alignment of SSU sequences of 27 acanthocephalan species, file name: Acan_18S_str_align_AEM2014.doc; online at http://folia.paru.cas.cz/ suppl/2014-4-377a.doc

The order Echinorhynchida includes 11 families, most species of which typically infect teleost fish, but have also been recorded from amphibians and reptiles (Amin 1987, 2013, Pichelin and Cribb 2001, Smales 2012). Familial diagnosis has been classically based on a unique combination of morphological characters rather than on shared derived features (García-Varela and Nadler 2005). However, recent studies using a combination of molecular and morphological data have demonstrated that several genera belonging to this order are in fact paraphyletic or polyphyletic (Monks 2001, García-Varela and Nadler 2005, Verweyen et al. 2011). This is not surprising since few stable characters have been identified as accurate markers for family-level identification in the taxonomy of acanthocephalans (Pichelin and Cribb 2001).

During a parasitological investigation of a pelagic marine fish, the round scad Decapterus punctatus $(\mathrm{Cu}-$ vier) (Carangidae), found in Brazilian coastal waters, several mature specimens of an echinorhynchid-like acanthocephalan species were recovered from the intestine. A morphological study of these specimens revealed that they could not be unequivocally assigned to any of the extant families recognised by Amin (1987). Indeed, these specimens resembled species of the genus Rhadinorhyn-

Address for correspondence: P.E. Braicovich, Laboratorio de Parasitología, Instituto de Investigaciones Marinas y Costeras (IIMyC), FCEyN, Universidad Nacional de Mar del Plata-CONICET, Funes 3350, (7600) Mar del Plata, Argentina. Phone: (+54) 223 475-4060; Fax: (+54) 223 475-3150; E-mail: braicovi@mdp.edu.ar 
chus Lühe, 1911 (Rhadinorhynchidae) in having dorsoventral asymmetry of the proboscis hooks, the presence of greatly enlarged hooks forming a ring at the base of the proboscis and four tubular cement-glands. However, the presence of an unarmed trunk suggests that these specimens do not belong to this genus. The combination of the unarmed trunk with a cylindrical proboscis and four tubular cement glands rather suggests that they could be placed into the genus Neorhadinorhynchus Yamaguti, 1939 (Cavisomidae). Nevertheless, basal hooks forming a ring around the base of the proboscis has only been described for only two species of the genus, namely N. aspinosus (Fukui et Morisita, 1937) and N. nudus (Harada, 1938) (reported as N. nudum by Amin and Nahhas 1994) and the dorsoventral asymmetry of the proboscis hooks displayed by the recovered specimens has not been described for species of Neorhadinorhynchus.

As this combination of morphological characteristics does not allow our specimens to be placed into either of the above mentioned families, we performed phylogenetic analyses in an attempt to clarify their taxonomic status. The genetic evidence strongly suggests that these organisms represent both a new genus and species of a new family of Echinorhynchida, which are described herein.

\section{MATERIALS AND METHODS}

\section{Sample collection and morphological study}

Adult acanthocephalans were recovered from the intestines of 18 round scad, Decapterus punctatus, caught off the coast of Cabo Frio, Brazil $\left(22^{\circ} 53\right.$ 'S; $\left.42^{\circ} 00^{\prime} \mathrm{W}\right)$ in November 2009. Parasites were removed and some specimens were relaxed in distilled water for several hours to allow extrusion of the proboscis, before being fixed in 5\% buffered formalin and stored in 70\% ethanol. Some specimens were washed in saline solution and then fixed in 95\% ethanol for use in molecular analysis.

Specimens used for microscopic study were cleared in lactic acid and examined as wet mounts. Others were stained with Semichon's aceto-carmine, cleared in methyl salicylate and mounted in Canada balsam. The descriptions and measurements are based on complete specimens with undamaged proboscides. Drawings were made with the aid of a drawing tube and all measurements are presented in millimetres unless otherwise indicated, with the mean value being followed by the observed range in parentheses.

\section{Molecular data and phylogenetic analyses}

Genomic DNA was extracted from individual specimens using the DNeasy Blood and Tissue ${ }^{\circledR}$ kit (QIAGEN, Hilden, Germany) according to the manufacturer's instructions. Two regions of nuclear ribosomal DNA (rDNA), the near-complete SSU rDNA ( 1 $600 \mathrm{bp})$ and LSU rDNA $(\sim 2600 \mathrm{bp})$, and a partial sequence $(\sim 660 \mathrm{bp})$ of the cytochrome $c$ oxidase subunit 1 gene $(\operatorname{cox} 1)$ were amplified using either the primers described by Folmer et al. (1994) and García-Varela and González-Oliver (2008), or their modifications (see primer sequences in Table 1). Each $50 \mu \mathrm{PCR}$ reaction consisted of $0.5 \mathrm{mM}$ of each primer, $10 \mu 1$ of $5 X$ Green Go Taq ${ }^{\circledR}$ Reaction Buffer, $200 \mathrm{mM}$ deoxynucleoside triphosphates, $1.5 \mathrm{mM} \mathrm{MgCl}_{2}$, and $1 \mathrm{U}$ taq polymerase
(GoTaq ${ }^{\circledR}$ DNA Polymerase, PROMEGA Corporation, Madison, USA). PCR cycling parameters included denaturation at $94^{\circ} \mathrm{C}$ for $3 \mathrm{~min}$, followed by 35 cycles of $94^{\circ} \mathrm{C}$ for $1 \mathrm{~min}$, annealing at $48-58^{\circ} \mathrm{C}$ (optimised for each gene region) for $1 \mathrm{~min}$, and extension at $72^{\circ} \mathrm{C}$ for $1 \mathrm{~min}$, followed by a post-amplification incubation at $72^{\circ} \mathrm{C}$ for $10 \mathrm{~min}$.

Each PCR product was purified using QIAquick spin columns (QIAquick Gel Extraction Kit, Qiagen). The fragments were sequenced for both DNA strands using the PCR primers. Sequencing was performed using Big Dye Terminator vs. 3.1 and 3130xl Genetic analyser (Applied Biosystem, Foster City, $\mathrm{CA}$ ) at the Genomic Unit, IB-INTA. Sequences were assembled and analysed with Vector NTI9.0.0. All sequences were deposited in the GenBank database (Table 2).

For phylogenetic analysis, the SSU, LSU and coxl gene sequences generated from the new taxon during the current study were aligned with those available for 23 other species representing 8 families of the order Echinorhynchida. Three species of the order Polymorphida, i.e. Corynosoma enhydri Morozov, 1940, Ibirhynchus dimorpha (Schmidt, 1973) and Southwellina hispida (Van Cleave, 1925), were used as outgroup according to García-Varela et al. (2013) (Table 2).

The alignment of the ribosomal sequences for phylogenetic study was performed according to secondary structure (Kjer et al. (2009). The structure alignment method provides an objective way to identify homologous positions within length heterogeneous sequences and to delimit regions of ambiguous alignment (the later to be excluded from analyses). In the present study the MUSCLE (Edgar 2004) procedure in MEGA v. 5.0 was used to generate a starting point alignment of the sequences. Then, they were manually aligned according to the predicted structure information of the rRNA gene (i.e. Gillespie et al. 2006), taking as base the annotated alignments of SSU and LSU sequences for animals recently provided by the empirical comparative studies of Marvaldi et al. (2009) and Mallatt et al. (2012). Sequences from the mitochondrial protein coding gene coxl were readily aligned based on their inferred (in silico-translated) cox 1 protein.

The data matrix for analyses with the combined data from the three markers contains 27 taxa, with 4534 characters of which 1571 were parsimony informative. Phylogenetic analyses were conducted using maximum parsimony (MP) with TNT (Goloboff et al. 2008) and with PAUP* (Swofford 1999), and also performed under maximum likelihood (ML) using PhyML 3.0 aLRT (Guindon and Gascuel 2003). Analyses were run on the combined data set of SSU, LSU and coxl, including all (27) terminal taxa, and also on each separate data set including the 27 taxa with data for SSU, 17 taxa with data for LSU and 22 taxa with data for coxl. Maximum parsimony analyses were performed using searches of 1000 random addition sequences and TBR branch swapping saving up to ten trees per replicate.

All characters had equal weights and gaps were treated as missing (this condition indicated to TNT, other programs treat gaps as missing by default). Bootstrap support values were obtained using TNT with resampling 1000 standard bootstrap replicates. Nodes with bootstrap values $\geq 50 \%$ were considered to be well-supported. For the ML analyses the original nexus file was executed in PAUP* and exported as a '.dat' file in Phylip format, then it was run using the program PhyML, under Substitution model: GTR, Gamma shape parameter: 0.543 , Number of categories: 4, Proportion of invariant: 0.290 . 
Table 1. Forward and reverse PCR primers for amplification and sequencing of Gymnorhadinorhynchus decapteri gen. n. et sp. n.

\begin{tabular}{lllc}
\hline Region & Primer name & Primer sequence forward and reverse 5'-3' & PCR product size (bp) \\
\hline SSU rDNA & fw.18IB & AGA TTA AGC CAT GCA TG & 1745 \\
& rev.lg18IB & CAA AGG GGG ACT TAA TC & - \\
LSU rDNA & fw1_28SIB & GGA GAG TGA ACA AGC CTG TG & 669 \\
& rev1_28SIB & CAG CAA CTA GAT GGT TCG ATT AG & - \\
& fw2_28SIB & ACC CGA AAG ATG GTG AAC TAT G & 1109 \\
& rv2_28SIB & CTT GGA GAC CTG TTG CGG & - \\
& fw3_28SIB & CCT GAA AAT GGA TGG CGC T & 1000 \\
& rv3_28S & GAT GTA CCG CCC CAG TCA AAC T & - \\
& fw28SIB & GGA AAG AAG ACC CTG TTG & 447 \\
& rv4_28SIB & CCA GCC AGT TAT CCC TGT & - \\
cox1 mDNA & fw.MitCox & AGT TCT AAT CAT AA(R) GAT AT(Y) GG & 660 \\
& rv.MitCox & TAA ACT TCA GGG TGA CCAAAA AAT CA ${ }^{\mathrm{b}}$ & - \\
\hline
\end{tabular}

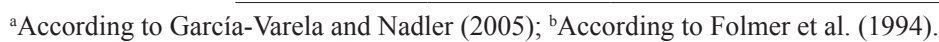

Table 2. Specimen information and GenBank accession numbers for taxa used in phylogenetic analysis.

\begin{tabular}{|c|c|c|c|c|c|}
\hline Species & Family & Host & $\begin{array}{l}\text { GenBank } \\
\text { Acc. No. SSU }\end{array}$ & $\begin{array}{l}\text { GenBank } \\
\text { Acc. No. LSU }\end{array}$ & $\begin{array}{l}\text { GenBank } \\
\text { Acc. No. cox } 1\end{array}$ \\
\hline \multicolumn{6}{|l|}{ Echinorhynchida } \\
\hline $\begin{array}{l}\text { Acanthocephaloides propinquus } \\
\text { (Dujardin, 1845) }\end{array}$ & Arhytmacanthidae & Gobius bucchichii Steindachner & AY830149 & AY829100 & DQ089713 \\
\hline Acanthocephalus dirus (Van Cleave, 1931) & Echinorhynchidae & Asellus aquaticus Linnaeus & AY830151 & AY829106 & DQ089718 \\
\hline Acanthocephalus lucii (Müller, 1776) & Echinorhynchidae & Perca fluviatilis (Linnaeus) & AY830152 & AY829101 & AM039837 \\
\hline Dentitruncus truttae Sinzar, 1955 & Illiosentidae & Salmo trutta Linnaeus & JX460866 & - & JX460903 \\
\hline Echinorhynchus gadi Zoega in Müller, 1776 & Echinorhynchidae & $\mathrm{NH}$ & AY218123 & - & AY218095 \\
\hline Echinorhynchus truttae Schrank, 1788 & Echinorhynchidae & Thymallus thymallus (Linnaeus) & AY830156 & AY829097 & DQ089710 \\
\hline Filisoma bucerium Van Cleave, 1940 & Cavisomidae & Kyposus elegans (Peters) & AF064814 & AY829110 & DQ089722 \\
\hline $\begin{array}{l}\text { Filisoma rizalinum } \\
\text { Tubangui et Masilungan, } 1946\end{array}$ & Cavisomidae & Scatophagus argus (Linnaeus) & JX014229 & - & - \\
\hline $\begin{array}{l}\text { Gorgorhynchoides bullocki } \\
\text { Cable et Mafarachisi, } 1970\end{array}$ & Isthmosacanthidae & Eugerres plumieri (Cuvier) & AY830154 & AY829103 & DQ089715 \\
\hline Gymnorhadinorhynchus decapteri & $\begin{array}{l}\text { Gymnorhadinor- } \\
\text { hynchidae }\end{array}$ & Decapterus punctatus (Cuvier) & KJ590123 & KJ590124 & KJ590125 \\
\hline Illiosentis sp. & Illiosentidae & $\mathrm{NH}$ & AY830158 & AY829092 & DQ089705 \\
\hline $\begin{array}{l}\text { Koronacantha mexicana } \\
\text { Monks et Pérez-Ponce de León, } 1996\end{array}$ & Illiosentidae & Pomadasys leuciscus (Günther) & AY830157 & AY829095 & DQ089708 \\
\hline $\begin{array}{l}\text { Koronacantha pectinaria } \\
\text { (Van Cleave, 1940) }\end{array}$ & Illiosentidae & $\begin{array}{l}\text { Microlepidotus brevipinnis } \\
\text { (Steindachner) }\end{array}$ & AF092433 & AY829094 & DQ089707 \\
\hline Leptorhynchoides thecatus (Linton, 1891) & Illiosentidae & Lepomis cyanellus (Rafinesque) & AK001840 & AY829093 & DQ089706 \\
\hline Pararhadinorhynchus sp. & Transvenidae & Siganus fuscescens (Houttuyn) & HM545903 & - & - \\
\hline $\begin{array}{l}\text { Pomphorhynchus bulbocoli } \\
\text { Linkins in Van Cleave, } 1919\end{array}$ & Pomphorhynchidae & $\begin{array}{l}\text { Lepomis macrochirus } \\
\text { Rafinesque }\end{array}$ & AF001841 & AY829096 & DQ089709 \\
\hline $\begin{array}{l}\text { Pomphorhynchus laevis } \\
\text { (Zoega in Müller, 1776) }\end{array}$ & Pomphorhynchidae & $\mathrm{NH}$ & AY423346 & - & AY218096 \\
\hline $\begin{array}{l}\text { Pomphorhynchus tereticollis } \\
\text { (Rudolphi, 1809) }\end{array}$ & Pomphorhynchidae & Platichthys flesus (Linnaeus) & AY423347 & - & JN695508 \\
\hline $\begin{array}{l}\text { Pseudoleptorhynchoides lamothei } \\
\text { Salgado-Maldonado, } 1976\end{array}$ & Illiosentidae & $\begin{array}{l}\text { Ariopsis guatemalensis } \\
\text { (Günther) }\end{array}$ & EU090950 & EU090951 & EU090949 \\
\hline $\begin{array}{l}\text { Rhadinorhynchus lintoni } \\
\text { Cable et Linderoth, } 1963\end{array}$ & Rhadinorhynchidae & $\begin{array}{l}\text { Selar crumenophthalmus } \\
\text { (Bloch) }\end{array}$ & JX014224 & - & - \\
\hline Rhadinorhynchus pristis (Rudolphi, 1802) & Rhadinorhynchidae & Gempylus serpens Cuvier & JX014226 & - & - \\
\hline Rhadinorhynchus sp. & Rhadinorhynchidae & Sciaenidae & AY062433 & AY829099 & DQ089712 \\
\hline Serrasentis sagittifer (Linton, 1889) & Rhadinorhynchidae & Johnius coitor (Hamilton) & JX014227 & - & - \\
\hline $\begin{array}{l}\text { Transvena annulospinosa } \\
\text { Pichelin et Cribb, } 2001\end{array}$ & Transvenidae & $\begin{array}{l}\text { Anampses neoguinaicus } \\
\text { Bleeker }\end{array}$ & AY830153 & AY829098 & DQ089711 \\
\hline \multicolumn{6}{|l|}{ Polymorphida (outgroup) } \\
\hline Corynosoma enhydri Morozov, 1940 & Polymorphidae & Enhydra lutris (Linnaeus) & GQ981436 & AY829107 & DQ089719 \\
\hline Ibirhynchus dimorpha (Schmidt, 1973) & Polymorphidae & Eudocimus albus (Linnaeus) & AF001837 & GQ981437 & GQ981438 \\
\hline Southwellina hispida (Van Cleave, 1925) & Polymorphidae & $\begin{array}{l}\text { Phalacrocorax brasilianus } \\
\text { (Gmelin) }\end{array}$ & JX014228 & EU267811 & HМ636469 \\
\hline
\end{tabular}

$\mathrm{NH}$ - host identity not provided in GenBank. 


\section{RESULTS}

Class Palaeacanthocephala Meyer, 1931

Order Echinorhynchida Southwell et MacFie, 1925

\section{Gymnorhadinorhynchidae fam. n.}

Diagnosis. Echinorhynchida. Trunk elongate, lacking spines. Neck present. Proboscis cylindrical; hooks arranged in longitudinal rows, basal hooks forming a circle at same level, abruptly larger than anterior hooks. Proboscis receptacle double-walled; ganglion about $1 / 2$ of proboscis receptacle. Lemnisci 2, variable in length. Testes 2. Cement glands 4, tubular. Seminal vesicle present. Eggs with polar elongation of E2 membrane. Parasites of marine fishes.

Type genus: Gymnorhadinorhynchus gen. n.

\section{Gymnorhadinorhynchus gen. n.}

Diagnosis. Gymnorhadinorhynchidae. Trunk cylindrical, dilated anteriorly. Proboscis hooks arranged in longitudinal rows, with differences between dorsal and ventral hooks, basal hooks arranged in circle, abruptly larger than anterior hooks. Lemnisci long, tubular, not extending beyond proboscis receptacle. Testes 2 , ovoid, in tandem. Cement glands 4, tubular, in 2 pairs, posterior pair overlapping slightly with anterior pair. Seminal vesicle present.

\section{Gymnorhadinorhynchus decapteri sp. n. Figs. 1-6}

Description (general description based on whole mounts of 11 males and 12 females; measurements based on 10 males and 10 females). Body cylindrical, moderate in size. Gravid females larger than males. Trunk thick-walled, unspined. Body wall with distinct nuclei. Proboscis relatively long, cylindrical, slightly curved and armed with 10 longitudinal rows of recurved hooks with simple shallow root structure; each row includes 22-26 hooks (measurements given in Table 3). Proboscis hooks decrease slightly in size from apex to base, apical hooks smaller than following ones. Ventral hooks with stronger curvature and thicker than dorsal hooks; basal hooks (= hook 1) considerably longer than those directly anterior to them, arranged at same level, forming a ring and projecting at right angle from proboscis. Neck short, slightly curved towards ventral side, with 1 pair of dorsal sensory pits. Lemnisci digitiform, shorter than proboscis receptacle. Proboscis receptacle double-walled. Ganglion near middle of proboscis receptacle.

Male (Figs. 1-3): Body 8.69 (6.82-10.90; $\mathrm{n}=10)$ long and $0.50(0.42-0.58 ; \mathrm{n}=10)$ maximum width. Proboscis $0.79(0.56-1.16 ; \mathrm{n}=9)$ long and $0.15(0.13-0.18 ; \mathrm{n}=10)$ wide. Neck $0.14(0.13-0.16 ; \mathrm{n}=8)$ in dorsal length. Proboscis receptacle $1.9(1.46-2.14 ; \mathrm{n}=4)$ long and 0.24 $(0.18-0.38 ; \mathrm{n}=4)$ wide. Lemnisci $1.23(0.80-1.65 ; \mathrm{n}=9)$ long and $0.08(0.04-0.11 ; \mathrm{n}=7)$ wide. Testes 2 , oval, in

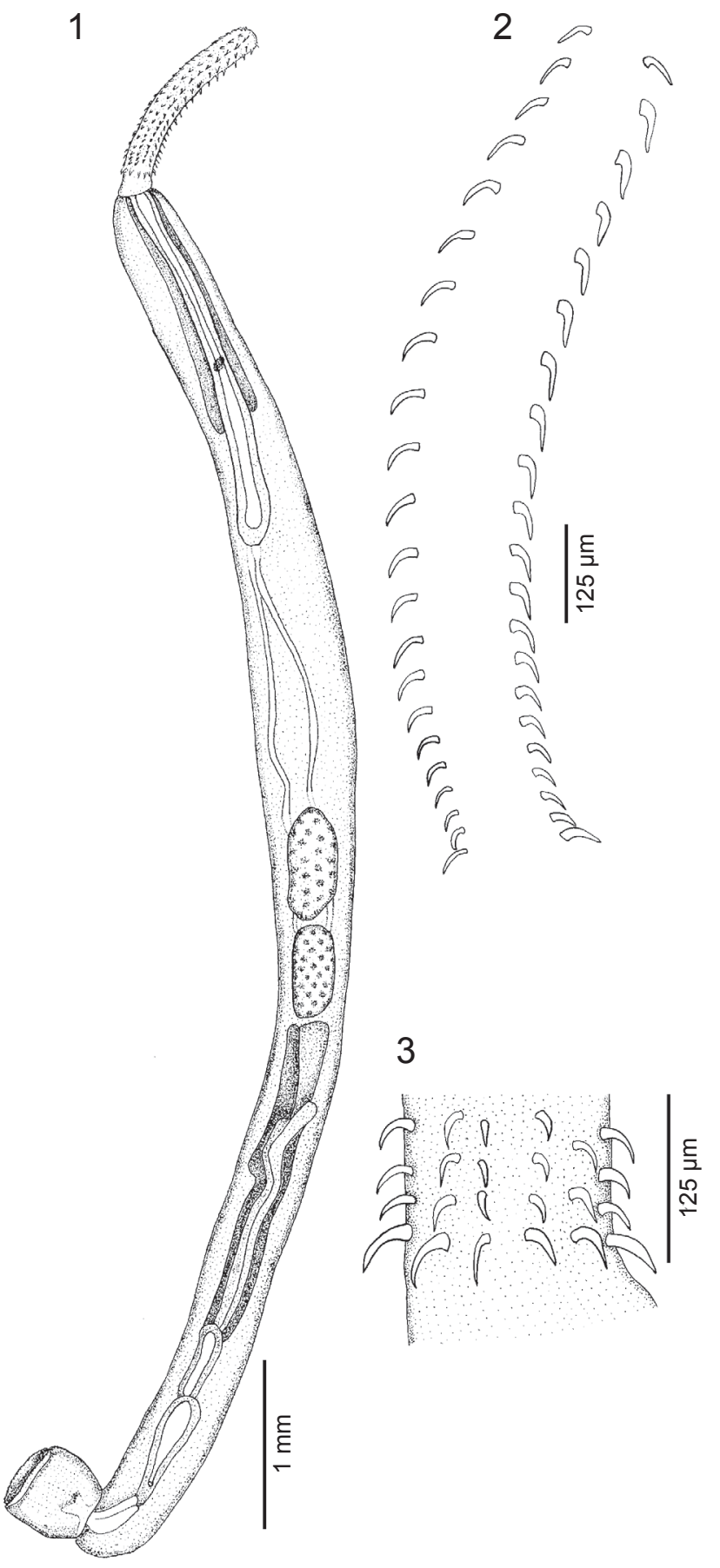

Figs. 1-3. Gymnorhadinorhynchus decapteri gen. n. et sp. n. from Decapterus punctatus. Fig. 1. Male, lateral view. Fig. 2. Male, proboscis showing dorsal and ventral rows of hooks. Fig. 3. Male, proboscis showing basal hooks.

tandem, contiguous; anterior testis 0.97 (0.57-1.70; $\mathrm{n}=10)$ long and $0.27(0.21-0.34 ; \mathrm{n}=10)$ wide; posterior testis $0.87(0.54-1.48)$ long and $0.25(0.21-0.29)$ wide. Cement glands 4, tubular; anterior pair reaching posterior testis, $1.95(1.40-3.30 ; \mathrm{n}=9)$ long and $0.13(0.10-0.17$; $\mathrm{n}=8)$ wide; posterior pair shorter, $1.10(0.49 .35-1.80$; $\mathrm{n}=8)$ long and $0.13(0.08-0.20 ; n=8)$ wide. Säfftigen's 
pouch surrounded by cement glands ducts. Seminal vesicle claviform, $0.72(\mathrm{n}=1)$ long. Copulatory bursa welldeveloped, hemispherical with scalloped edge and internal sensory papillae, $0.56(0.35-0.85 ; \mathrm{n}=4)$ long and $0.56(0.52-0.61 ; \mathrm{n}=4)$ wide, when everted. Genital pore subterminal.

Female (Figs. 4-6): Body $19.11(12.96-28.70 ; n=10)$ long and $0.60(0.42-0.90 ; \mathrm{n}=10)$ greatest width. Proboscis $1.1(0.72-1.50 ; \mathrm{n}=9)$ long and $0.16(0.14-0.17$; $\mathrm{n}=9)$ wide. Neck $0.17(0.15-0.19 ; \mathrm{n}=7)$ in dorsal length. Proboscis receptacle $2.39(2.18-2.60 ; \mathrm{n}=4)$ long and 0.81 $(0.13-2.42 ; \mathrm{n}=4)$ wide. Lemnisci $1.51(1.03-2.22 ; \mathrm{n}=7)$ long and $0.13(0.04-0.25 ; \mathrm{n}=7)$ wide. Female reproductive system $(\mathrm{n}=3)$ : uterine bell and selector apparatus 0.33 (0.23-0.41) long; uterus tubular 4.12 (3.31-4.60) long; vaginal sphincter present; vagina $0.16(0.13-0.19)$ long. Eggs fusiform, $0.049(0.045-0.050 ; n=10)$ long and $0.013(0.010-0.015 ; \mathrm{n}=10)$ wide.

Type host: Decapterus punctatus (Cuvier) (Actinopterygii: Carangidae).

Ty p e 1 o c a lity: Cabo Frio, Rio de Janeiro, Brazil (22 $53^{\circ} \mathrm{S}$; $\left.42^{\circ} 00^{\prime} \mathrm{W}\right)$.

Site: Intestine.

Prevalence : $78 \%$ (14 out of 18 hosts examined).

Mean intensity (range): 10.9 (1-64).

Type material: Holotype No. 35941a (male), allotype No. 35941 b (female), and paratypes ( 1 male and 2 females) No. 35941 d-e were deposited in the Coleçao Helmintologica do Instituto Oswaldo Cruz (CHIOC), Rio de Janeiro, Brazil. Two additional paratypes are deposited in the Institute of Parasitology, České Budějovice, Czech Republic (IPCAS A-85).

New sequences: Table 2.

Et y mology: The family and genus names are a combination of the Greek word gymnos (naked) and Rhadinorhynchidae/ Rhadinorhynchus, a family/genus of similar morphology, but lacking somatic spines. The specific epithet refers to the genus (Decapterus) of the type host.

\section{Phylogenetic analyses}

Structural alignments of ribosomal markers. Multiple sequence alignments of nearly complete SSU and LSU were generated across 27 and 17 acanthocephalan taxa, respectively. This structure-based alignment is in the nexus file (see S1). The aligned sequences of the SSU (small subunit) are interleaved in blocks each starting with a mask with the annotated structure symbols (as in the study by Marvaldi et al. 2009). The alignment of sequences of the LSU (large subunit) is not structure-annotated but numbers in the mask indicate the corresponding block in the data presented by Mallatt et al. (2012) where the annotated structure of the LSU can be tracked. The study by Mallatt et al. (2012) includes four acanthocephalans amongst which Leptorhynchoides thecatus (Linton, 1891) is in common with the present study. Because of the im-
4
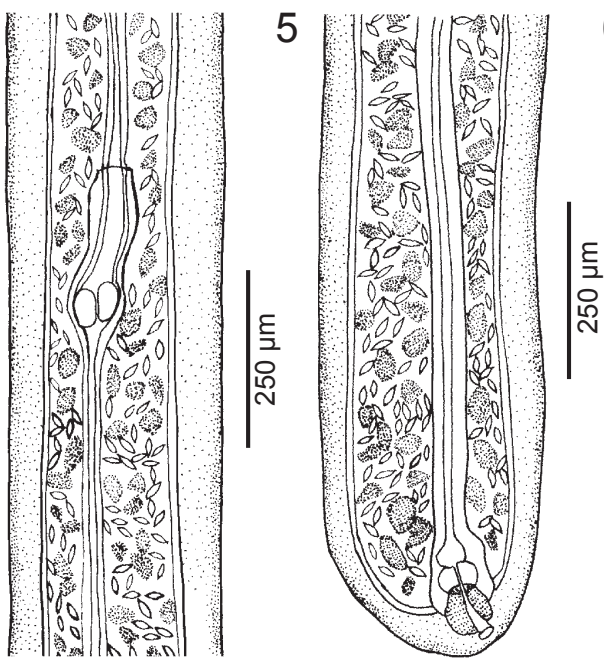

6

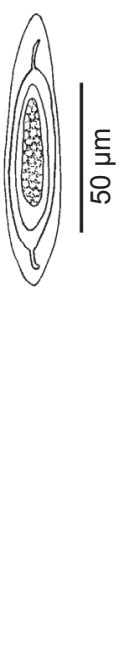

Figs. 4-6. Gymnorhadinorhynchus decapteri gen. n. et sp. n. from Decapterus punctatus. Figs. 4, 5. Female, reproductive system. Fig. 6. Egg.

portance of the SSU as a phylogenetic marker for studies on acanthocephalans (and many other groups), the annotated alignment generated in the present study for the 27 acanthoceplalan taxa is also provided in a Word colour file to serve as alignment template for future studies (see S2) .

Echinorhynchid phylogeny and the placement of the new taxon. Maximum parsimony analysis of the combined SSU, LSU and cox 1 data resulted in two trees of 5355 steps in length, the strict consensus of which is presented in Fig. 7. The tree obtained using ML is highly concordant in topology to the one obtained using the maximum parsimony method (Fig. 7).

The phylogenetic hypothesis (Fig. 7) indicates a monophyletic order Echinorhynchida which contains six main, well supported clades. The new taxon Gymnorhadinorhynchus decapteri sp. n., clusters into a distinct, well supported clade with three other taxa: an unidentified species from Mexico (originally named 'Rhadinorhynchus sp.') and a further two species that belong to two genera from the family Transvenidae, Transvena annulospinosa Pichelin et Cribb, 2001 and Pararhadinorhynchus sp.

Based upon the results from both the combined and individual sequence data analyses, G. decapteri sp. n., and the unidentified Mexican species appear to be most closely related inferred from a significant bootstrap support. Examination of the apomorphy list for the node of these two species indicates that they have more than 80 synapomorphies supporting this relationship, including 18 unique changes: 5 from the SSU, 11 from the LSU and 2 from the coxl gene. Two species belonging to the genus Rhadinorhynchus, R. lintoni Cable et Linderoth, 1963 and $R$. pristis (Rudolphi, 1802), clustered with members of the Pomphorhynchidae to constitute another well supported clade that is distant from the gymnorhadinorhynchids. Furthermore, two representatives of the Cavi- 
Table 3. Measurements (in micrometres) of dorsal and ventral proboscis hooks (length $\times$ width) of males and females of Gymnorhadinorhynchus decapteri gen. $\mathrm{n}$. et sp. $\mathrm{n}$.

\begin{tabular}{|c|c|c|c|c|}
\hline \multirow{2}{*}{$\begin{array}{l}\text { Hook no. } \\
\text { (from } \\
\text { anterior) }\end{array}$} & \multicolumn{2}{|c|}{ Male } & \multicolumn{2}{|c|}{ Female } \\
\hline & Dorsal hooks & Ventral hooks & Dorsal hooks & Ventral hooks \\
\hline 1 & $43 \times 10$ & $53 \times 10$ & $45 \times 10$ & $63 \times 13$ \\
\hline 2 & $38 \times 10$ & $40 \times 10$ & $38 \times 10$ & $40 \times 10$ \\
\hline 3 & $40 \times 08$ & $43 \times 10$ & $35 \times 08$ & $43 \times 10$ \\
\hline 4 & $38 \times 08$ & $40 \times 10$ & $43 \times 08$ & $45 \times 10$ \\
\hline 5 & $43 \times 10$ & $40 \times 10$ & $43 \times 10$ & $53 \times 13$ \\
\hline 6 & $43 \times 10$ & $45 \times 13$ & $48 \times 10$ & $53 \times 13$ \\
\hline 7 & $45 \times 10$ & $50 \times 13$ & $45 \times 10$ & $55 \times 13$ \\
\hline 8 & $50 \times 10$ & $63 \times 13$ & $53 \times 10$ & $55 \times 13$ \\
\hline 9 & $53 \times 10$ & $65 \times 15$ & $50 \times 13$ & $55 \times 13$ \\
\hline 10 & $55 \times 13$ & $65 \times 15$ & $53 \times 13$ & $60 \times 15$ \\
\hline 11 & $55 \times 13$ & $65 \times 15$ & $53 \times 13$ & $63 \times 15$ \\
\hline 12 & $58 \times 13$ & $70 \times 15$ & $58 \times 13$ & $63 \times 15$ \\
\hline 13 & $55 \times 13$ & $68 \times 15$ & $58 \times 13$ & $63 \times 18$ \\
\hline 14 & $55 \times 13$ & $68 \times 15$ & $58 \times 13$ & $63 \times 18$ \\
\hline 15 & $55 \times 10$ & $70 \times 18$ & $58 \times 13$ & $68 \times 18$ \\
\hline 16 & $58 \times 10$ & $68 \times 18$ & $58 \times 13$ & $70 \times 18$ \\
\hline 17 & $60 \times 10$ & $70 \times 18$ & $58 \times 13$ & $68 \times 18$ \\
\hline 18 & $58 \times 10$ & $70 \times 18$ & $60 \times 13$ & $65 \times 20$ \\
\hline 19 & $60 \times 10$ & $70 \times 18$ & $58 \times 13$ & $68 \times 18$ \\
\hline 20 & $53 \times 10$ & $68 \times 18$ & $55 \times 13$ & $70 \times 20$ \\
\hline 21 & $55 \times 10$ & $45 \times 08$ & $53 \times 10$ & $70 \times 20$ \\
\hline 22 & $53 \times 10$ & - & $53 \times 10$ & $73 \times 23$ \\
\hline 23 & - & - & $55 \times 13$ & $70 \times 23$ \\
\hline 24 & - & - & $50 \times 08$ & $65 \times 20$ \\
\hline
\end{tabular}

somidae (in paraphyletic sequence) together with a representative of Arhytmacanthidae and two members of the genus Acanthocephalus Koelreuter, 1771 from the family Echinorhynchidae also formed a clade distant from the newly recognised taxon.

Representative members of some families are dispersed among different clades, rendering the families Rhadinorhynchidae, Pomphorhynchidae and Echinorhynchidae polyphyletic.

\section{DISCUSSION}

Based on the morphological and molecular criteria obtained during the current study, the new family Gymnorhadinorhynchidae is unequivocally a member of the order Echinorhynchida (Amin 1987, García-Varela and Nadler 2005, Verweyen et al. 2011). Since acanthocephalans have few stable morphological characters that can be considered useful for high level taxonomic classification, the families that belong to this order are typically defined by a combination of characters that, as stated by Pichelin and Cribb (2001), are in some cases interpreted erroneously. Indeed, several previously described echinorhynchid genera have been reassigned to families other than those in the classification provided by Amin (1987).

In a recent revision regarding the classification of the Acanthocephala, Amin (2013) agreed that the criteria for classifying the families of the Palaeacanthocephala based on morphology may need to be re-evaluated using gene sequencing methods. Amin (2013), however, did not accept the reassignment of some genera based on molecular evidence (García-Varela and Nadler 2005), as well as on morphological evidence such as the number of cement glands as a constant feature at the family level (Pichelin and Cribb 2001, Smales 2012), even when those genera are shown to be paraphyletic or polyphyletic in molecular phylogenetic studies.

Here, the morphological features described for Gymnorhadinorhynchus gen. n., such as the arrangement of the proboscis hooks and the presence of four cement glands, are consistent with the genus Rhadinorhynchus (Rhadinorhynchidae), whereas the absence of trunk spines places the specimens closer to the genus Neorhadinorhynchus (Cavisomidae). Based on morphology alone, however, this combination of characters precludes the inclusion of Gymnorhadinorhynchus gen. n. in either the Rhadinorhynchidae or the Cavisomidae. The new family can therefore be distinguished from the Rhadinorhynchidae by the absence of trunk spines and from the Cavisomidae by the difference in size between the dorsal and ventral proboscis hooks and also by the presence of specialised basal hooks arranged in a ring on the proboscis.

To reinforce the morphological differentiation specified here, molecular data have indicated Gymnorhadinorhynchus decapteri gen. $\mathrm{n}$. et $\mathrm{sp}$. $\mathrm{n}$. to be of a distinct lineage from valid members of the Rhadinorhynchidae, namely Rhadinorhynchus pristis and R. lintoni, and from the Cavisomidae, represented here by Filisoma bucerium Van Cleave, 1940 and F. rizalinum Tubangui et Masilungan, 1946. The apparent exception appears to be the undescribed Mexican species, which was originally reported as Rhadinorhynchus sp. by García-Varela et al. (2002), and which forms a sister taxon with $G$. decaperi. Although this Mexican species was originally named Rhadinorhynchus sp., results of the present study clearly suggest that it does not belong to Rhadinorhynchus, but may in fact represent a new genus within this newly erected family, which is closely related to Gymnorhadinorhynchus. Unfortunately, there was no material of this species available for comparison, which impeded the opportunity to conduct a morphological comparison with our specimens and to examine their possible generic identity.

The family Transvenidae contains two species that clustered together with the new taxon and it is the only family that possesses two cement glands. This family was erected by Pichelin and Cribb (2001) to accommodate two new genera Transvena Pichelin et Cribb, 2001 and Trajetura Pichelin et Cribb, 2001 together with members of Pararhadinorhynchus Johnston et Edmons, 1947. The inclusion of Pararhadinorhynchus in the Trasvenidae was not accepted by Amin (2013), but it is supported by the present molecular phylogenetic analyses. The genera belonging to this family can be clearly distinguished from Gymnorhadinorhynchus in having a claviform proboscis with hooks that decrease in length from the apex to the 

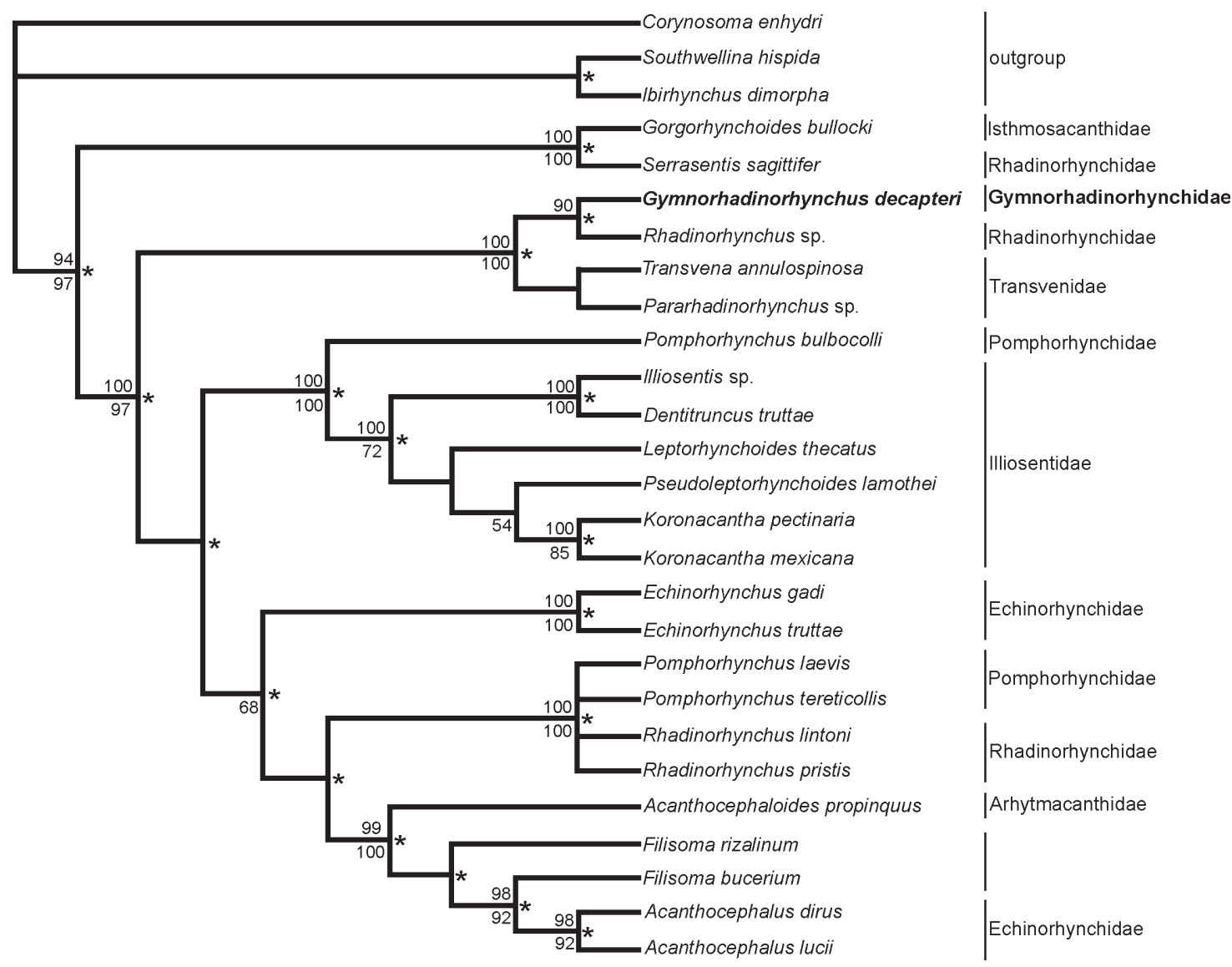

Fig. 7. Phylogenetic relationships of Echinorhynchida based on the parsimony analysis of the combined SSU, LSU and cox1 sequences. Strict consensus of the two most parsimonious trees of the length of 5355. Numbers above branches indicate bootstrap support values $\geq 50 \%$ from the combined data set, those below branches are those from separate SSU data. Nodes also recovered by maximum likelihood analysis are marked with asterisk. The families are indicated in the right side.

base and by the presence of two cement glands. In particular, Gymnorhadinorhynchus can be easily distinguished from the genus Transvena, in which the species possess trunk spines that are arranged in a single ring.

This combination of asymmetry in the proboscis hooks, the presence of the greatly enlarged spines forming a basal ring in the proboscis, the four tubular cement glands, and the molecular evidence, which has placed Gymnorhadinorhynchus into a separate clade from those families that share some of these features, justify the erection of Gymnorhadinorhynchidae as a new family in the order Echinorhynchida.

The presence of trunk spines is typically regarded to be a valuable taxonomic character for the Echinorhynchida, but it has been suggested by Pichelin et al. (2002) that spines can be easily lost or overlooked during morphological examination. In the present study more than 160 adult specimens were examined but trunk spines were not observed in any of the specimens, confirming that the absence of trunk spines is a valid diagnostic character for the new taxon.

Pichelin and Cribb (2001) also emphasised the importance of using the number and structure of cement glands as a useful family-level taxonomic feature for the Echinorhynchida. The importance of this character was later supported by phylogenetic studies that took both molecular and morphological approaches (García-Varela and González-Oliver 2008, Smales 2012), but were considered by Amin (2013). However, despite the diagnostic validity of using the number of cement glands at the family level, molecular data have demonstrated that families with the same number of cement glands may not necessarily be closely related. In fact, molecular analysis places the Cavisomidae and the Rhadinorhynchidae, both possessing four cement glands, into two separates clades. Furthermore, the Gymnorhadinorhynchidae also possesses four cement glands, but this family clusters more closely with the Transvenidae, the species of which possess only two cement glands. These data suggest that additional features, such as the presence or absence of trunk spines, must also be considered when attempting to morphologically discriminate echinorhynchid families.

Although the primary aim of this study was not to assess the current classification of the echinorhynchid genera and families or to call for their possible reorganisation, the molecular phylogenetic hypothesis presented 
here does highlight the existence of problems within the current classification scheme for the Acanthocephala. As already recommended by previous authors (García-Varela and Nadler 2005, Verweyen et al. 2011), the examination of further samples representing additional species within these groups is required to determine the existence of any meaningful feature that may be useful for discrimination at high level taxonomy. Future studies that combine both molecular and morphological approach in combination with ecological, life-history and behavioural data should provide a much more robust hypothesis concerning the relationships among members of this relatively understudied group of parasites. Finally, the comparative approach undertaken in the present study with regards to the ribosomal sequences enabled the construction of a structural alignment of the SSU and LSU rDNA markers for a wide sam- ple of echinorhynchid acanthocephalans. It is anticipated that this alignment will be useful as a homology template for future comparative phylogenetic studies that involve acanthocephalans and relatives in the Tree of Life.

Acknowledgements. Financial support was provided by grants from Consejo Nacional de Investigaciones Científicas y Técnicas (PIP No. 112-201101-00036); Agencia Nacional de Promoción Científica yTecnológica (PICT 2012 No. 2094) and Universidad Nacional de Mar del Plata (EXA 621/12). José L. Luque was supported by research fellowship from Conselho Nacional de Pesquisa e Desenvolvimento Tecnológico Brazil (CNPq). We wish to thank Aldenice Pereira for collection of additional samples for molecular studies. We are grateful to J. Daniel McLaughlin, Angela Rose Lapierre and Kellyanne Boyce for their helpful comments.

\section{REFERENCES}

Amin O.M. 1987: Key to the families and subfamilies of Acanthocephala with the erection of a new class (Polyacanthocephala) and a new order (Polyacanthorhynchida). J. Parasitol. 73: 1216-1219.

Amin O.M. 2013: Classification of the Acanthocephala. Folia Parasitol. 60: 273-305.

Amin O.M., Nahhas F.M. 1994: Acanthocephala of marine fishes off Fiji Islands, with descriptions of Filisoma longcementglandatus n. sp., Neorhadinorhynchus macrospinosus n. sp. (Cavisomidae) and gravid females of Rhadinorhynchus johnstoni (Rhadinorhynchidae); and keys to species of the genera Filisoma and Neorhadinorhynchus. J. Parasitol. 80: 768-774.

EDGAR R.C. 2004: MUSCLE: multiple sequence alignment with high accuracy and high throughput. Nucl. Acids Res. 32: $1792-$ 1797.

Folmer O., Black M., Hoeh W., Lutz R., Vrijenhoek R. 1994: DNA primers for amplification of mitochondrial cytochrome c oxidase subunit I from diverse metazoan invertebrates. Mol. Mar. Biol. Biotech. 3: 294-299.

García-Varela M., Cummings M.P., Pérez-Ponce de León G., Gardner S.L., Laclettea J.P. 2002: Phylogenetic analysis based on 18S ribosomal RNA gene sequences supports the existence of class Polyacanthocephala (Acanthocephala). Mol. Phylogenet. Evol. 23: 288-292.

García-VArela M., González-Oliver A. 2008: The systematic position of Leptorhynchoides (Kostylew, 1924) and Pseudoleptorhynchoides (Salgado-Maldonado, 1976), inferred from nuclear and mitochondrial DNA gene sequences. J Parasitol. 94: 959-962.

García-VArela M., Nadler S.A. 2005: Phylogenetic relationships of Palaecanthocephala (Acanthocephala) inferred from SSU and LSU rDNA gene sequences. J. Parasitol. 91: 14011409

García-Varela M., Pérez-Ponce de León G., Aznar F.J., NADLER S.A. 2013: Phylogenetic relationship among genera of Polymorphidae (Acanthocephala), inferred from nuclear and mitochondrial gene sequences. Mol. Phylogenet. Evol. 68: 176-84.

Gillespie J.J., Johnston J.S., Cannone J.J., Gutell R.R. 2006: Characteristics of nuclear $(18 \mathrm{~S}, 5.8 \mathrm{~S}, 28 \mathrm{~S}$ and $5 \mathrm{~S})$ and mitochondrial (12S and 16S) rRNA genes of Apis mellifera (In- secta: Hymenoptera): structure, organization, and retrotransposable elements. Insect Mol. Biol. 15: 657-686.

Goloboff P.A., FARris J.S., Nixon K.C. 2008: TNT, a free program for phylogenetic analysis. Cladistics 24: 774-786.

Guindon S., Gascuel O. 2003: A simple, fast, and accurate algorithm to estimate large phylogenies by maximum likelihood. Syst. Biol. 52: 696-704.

Kjer K.M., Roshan U., Gillespie J.J. 2009: Structural and evolutionary considerations for multiple sequence alignment of RNA, and the challenges for algorithms that ignore them. In: M.S. Rosenberg (Ed.), Sequence Alignment: Methods, Models, Concepts, and Strategies. University of California Press, Berkeley, CA, pp. 105-149.

Mallatt J., Craig C.W., Yoder M.J. 2012: Nearly complete rRNA genes from 371 Animalia: updated structure-based alignment and detailed phylogenetic analysis. Mol. Phylogenet. Evol. 64: 603-617.

Marvaldi A.E., Duckett C.N., Kuer K.M., Gillespie J. 2009: Structural alignment of $18 \mathrm{~S}$ and $28 \mathrm{~S}$ rDNA sequences provides insights into phylogeny of Phytophaga (Coleoptera: Curculionoidea and Chrysomeloidea). Zool. Scri. 38: 63-77.

Monks S. 2001: Phylogeny of the Acanthocephala based on morphological characters. Syst. Parasitol. 48: 81-116.

Pichelin S., Cribb T.H. 2001: The status of the Diplosentidae (Acanthocephala: Palaeacanthocephala) and a new family of acanthocephalans from Australian wrasses (Pisces: Labridae). Folia Parasitol. 48: 289-303.

Pichelin S., Smales L.R., Bray R.A. 2002: A discussion on the Heteracanthocephalidae Petrochenko, 1956 (Acanthocephala: Palaeacanthocephala). Syst. Parasitol. 52: 145-152.

Smales L.R. 2012: A new acanthocephalan family, the Isthmosacanthidae (Acanthocephala: Echinorhynchida), with the description of Isthmosacanthus fitzroyensis n. g., n. sp. from threadfin fishes (Polynemidae) of northern Australia. Syst. Parasitol. 82: 105-111.

SwofFord D.L. 1999: 'PAUP*: Phylogenetic Analysis Using parsimony (* and other methods), ver. 4.0'. Sinauer: Sunderland, MA, USA.

Verweyen L., Klimpel S., Palm H.W. 2011: Molecular phylogeny of the Acanthocephala (class Palaeacanthocephala) with a paraphyletic assemblage of the orders Polymorphida and Echinorhynchida. PLoS ONE 6: e28285. 